\title{
A Leukocyte Detection System Using Scale Invariant Feature Transform Method
}

\author{
Lina Arlends Chris, Bagus Mulyawan, and Agus Budi Dharmawan
}

\begin{abstract}
This paper describes an automatic detection and recognition system of leukocytes on a given microscopic image. The developed system detects the locations of leukocytes from a blood cell image. After the automatic detection, the system classifies each leukocyte in one of the five categories (neutrophils, eosinophils, basophils, lymphocytes, and monocytes). The system processes an input image with the Scale Invariant Feature Transform (SIFT) algorithm for leukocyte detection. Meanwhile, two different recognition methods, i.e. the Euclidean distance and the Co-occurrence matrix methods are applied for automatic recognition. The combination of detection and recognition approaches provides the optimal recognition accuracies for almost all leukocyte types.
\end{abstract}

Index Terms-Leukocyte detection, leukocyte recognition, microscopic image, scale invariant feature transform.

\section{INTRODUCTION}

Blood is a bodily fluid that delivers nutrients and oxygen to cells. The analysis of blood cells can be used to detect blood disorder or to determine the presence of infectious diseases in human body. In order to identify the hematopoietic system disorders, hematologists need to perform the blood cells identification and counting for every blood elements, such as the erythrocytes (red cells), leukocytes (white cells), and platelets [1]. Since the task is very tedious and really time consuming, an automaticblood detection, recognition, and counting system is necessary and helpful.

Several researchers have proposed various methods todetect and recognize the blood cells, such as the work by Markiewicz using the Support Vector Machine method [2], Colunga with EMalgorithm [3], and Neural Network-based classifiers [4], [5]. However, the detection and recognition systems have not been tested for blood cells that were influenced by rotation or illumination effects after the segmentation process.

In this paper, an automatic leukocyte detection system that can detect the white blood cell locations from microscopic images is developed. The proposed system works based on the Scale Invariant Feature Transform (SIFT) method. First, the system detects the white blood cells locations using the SIFT method, then the system crops the images which contains the region of interest. After the automatic detection

Manuscript received August 7, 2014; revised November 13, 2014. This work was supported in part by the Indonesian Directorate General of Higher Education under Hibah Penelitian Unggulan Perguruan Tinggi Tahun Anggaran 2014.

Lina A. Chris, B. Mulyawan, and A. B. Dharmawan are with the Computer Science Department, Faculty of Information Technology, Tarumanagara University, Jl. Letjen. S. Parman 1, Jakarta 11440, Indonesia (e-mail: lina@untar.ac.id). and cropping, the system will recognize the leukocyte type using two different recognition methods: 1) the distance based recognition system using the Euclidean distance method, and 2) the color based recognition system using the co-occurrence matrix method.

The remainder of this paper is organized as follows. In Section II, the proposed leukocyte detection system based on SIFT algorithmis explained. Section III presents the leukocyte recognition system, while Section IV describes the experimental setup and results. Finally, the conclusion is presented in Section V.

\section{LEUKOCYTE Detection SySTEM}

In the proposed system, the Scale Invariant Feature Transform (SIFT) method is applied to detect the leukocytes from the captured microscopic images. The SIFT algorithm, developed by Lowe [6]-[8] is an algorithm for image features generation which are invariant to image translation, scaling, rotation and partially invariant to illumination changes and affine projection [9]. The steps for defining the SIFT image features are as follows: 1) Scale space construction, 2) Keypoint localization, 3) Orientation assignment, 4) Keypoint descriptor.

First, the system creates a scale space from the input imagesby calculating the Difference of Gaussian (DoG) using the Gaussian kernel. This step is necessary as an input image may consist unncessary details for detection or recognition processes. Therefore it is important to identify locations and scales that contains only the region of interest from an image. The first step for detecting locations that are invariant to scale changes is by constructing a continuous function of scale, known as the scale space. The scale space of an image is defined as a function, $L(x, y, \sigma)$, that is produced from the convolution of a variable-scale Gaussian, $G(x, y, \sigma)$, with an input image $I(x, y)$

$$
L(x, y, \sigma)=G(x, y, \sigma) * I(x, y)
$$

where * is the convolution operation in $x$ and $y$, and

$$
G(x, y, \sigma)=\left(1 / 2 \pi \sigma^{2}\right) e^{-\left(x^{2}+y^{2}\right) / 2 \sigma^{2}}
$$

To build the DoG pyramid, the input image is convolved iteratively with the Gaussian kernel. The last convolved image is down-sampled in each image direction by factor of 2 , and the convolving process is repeated [9]. Each collection of images of the same size are then build together the so-called Gaussian pyramid, which is represented by a $3 \mathrm{D}$ function defined in Eq. 1. The DoG pyramid is computed from the difference of each two nearby images in Gaussian pyramid. Fig. 1 shows the constructed scale space images. 


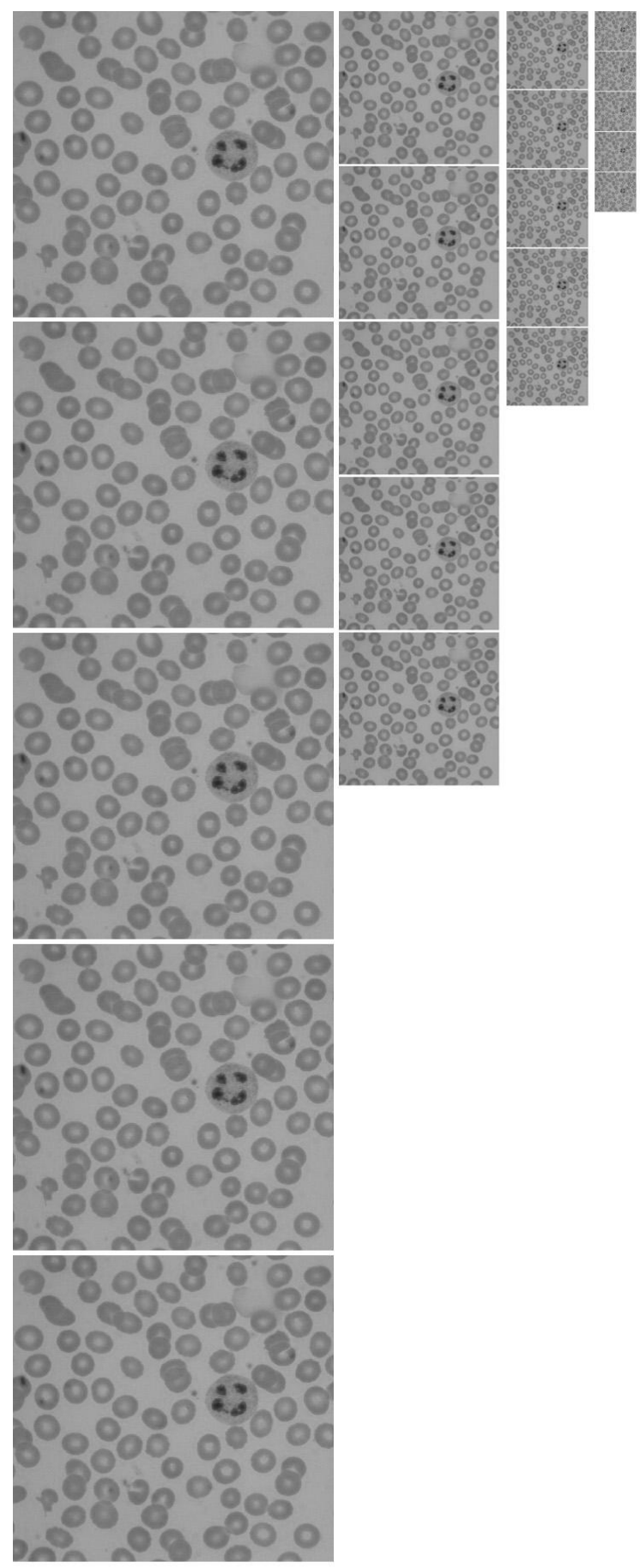

Fig. 1. The scale space images for leukocyte detection system.

The next step is to define keypoints. Keypoints are pixels from an image which have constant values for scaling, rotation, blurring, and illumination changes. Keypoint construction is done by finding the local extrema (maxima or minima) of DoG function. The local extrema are detected by comparing each pixel with its neighbors in the scale space. If the pixel value is higher or lower than the maxima or minima, then the pixel becomes the candidate for being a keypoint. The DoG function will have a strong response along edges, even if the location along the edge is poorly determined and therefore unstable to small amounts of noise.

The next step is to localize keypoints. In keypoint localization, a threshold cutting is applied on simple contrast value for each keypoint. The low contrast feature points are generally less reliable than high contrast feature points. The keypoints are selected only if they are larger than all of these neighbors or smaller than all of them. To improve the stability of matching, the points that have low contrast or are poorly localized along an edge are rejected.

After the thresholding step, the system performs the corner detection process. Harris corner detection algorithm is realized by calculating each pixel's gradient [10]. If the absolute gradient values in two directions are both great, then judge the pixel as a corner. Harris corner detector is defined as follows [11]:

$$
\begin{gathered}
R=\operatorname{det} M-k(\text { trace } M)^{2} \\
M(x, y)=\left[\begin{array}{cc}
I_{u}^{2}(x, y) & I_{u v}(x, y) \\
I_{u v}(x, y) & I_{v}^{2}(x, y)
\end{array}\right] \\
I_{u}^{2}(x, y)=X^{2} \otimes \omega(x, y) \\
I_{v}^{2}(x, y)=Y^{2} \otimes \omega(x, y) \\
I_{u v}(x, y)=X Y \otimes \omega(x, y) \\
\omega(x, y)=\frac{1}{2 \pi} e^{-\frac{x^{2}+y^{2}}{2 \sigma^{2}}}
\end{gathered}
$$

where $k$ is an empirical value; $\omega(x, y)$ is a Gaussian function; $I_{u}(x, y)$ and $I_{v}(x, y)$ are the partial derivatives of the gray scale in direction $u$ and $v$ at point $\otimes$, and $I_{u v}(x, y)$ is the second-order mixed partial derivative; $X$ and $Y$ are the first-order directional differentials, which can be approximately calculated by convolving the gray scale and difference operators in direction $u$ and $v ; \otimes$ refers to convolution. If $R$ exceeds certain threshold, then the point is determined as a corner.

Once the SIFT feature location is determined, a main orientation is assigned to each feature based on local image gradients. For each pixel of the region around the feature location the gradient magnitude and orientation are computed respectively as [9]:

$m(x, y)=\sqrt{(L(x+1, y, \sigma)-L(x-1, y, \sigma))^{2}+(L(x, y+1, \sigma)-L(x, y-1, \sigma))^{2}}$

$\theta(x, y)=\arctan ((L(x, y+1, \sigma)-L(x, y-1, \sigma)) /(L(x+1, y, \sigma)-L(x-1, y, \sigma)))$

Finally, the region around a keypoint is divided into $4 \times 4$ boxes. The gradient magnitudes and orientations within each box are computed and weighted by appropriate Gaussian window, and the coordinate of each pixel and its gradient orientation are rotated relative to the keypoints orientation. Then, for each box an 8 bins orientation histogram is established. From the 16 obtained orientation histograms, a 128 dimensional vector (SIFT-descriptor) is built.

\section{LEUKOCYTE RECOGNITION SySTEM}

For the recognition system, two methods are applied to the system: 1) the distance based recognition system using the 
Euclidean distance method, and 2) the color based recognition system using the co-occurrence matrix method.

In the Euclidean distance based recognition system, the dissimilarities between the testing and training feature vectors are calculated using the Euclidean distance measurement [12], [13]:

$$
r=\|\mathbf{x}-\mathbf{w} \quad\|
$$

With $r$ is the Euclidean distance between $\mathbf{x}$ as the testing feature vector and $\mathbf{w}$ as the training feature vector. A small $r$ value indicates a high similarity of two images.

Meanwhile, in the color based recognition system using the co-occurrence matrix method, the co-occurrence matrix is constructed by clustering the gray-scale values of an image. Such matrix is derived from the angular relationship between the neighboring pixels as well as the distances between them. The higher the color intensity of an image, the larger size of co-occurrence matrix can be obtained. First, the probability value $p(i, j)$ of the color frequency $f(i, j)$ of index pair $i$ and $j$ is calculated by [12], [13]:

$$
p(i, j)=\frac{1}{\sum f(i, j)} \times\left[\begin{array}{ccc}
f(i, j) & f(i, j) & \ldots . . \\
f(i, j) & f(i, j) & \ldots . . \\
\ldots \ldots . . & \ldots \ldots . . & \ldots \ldots .
\end{array}\right]
$$

Next, obtainthe Haralick features by processing the probability values of the co-occurrence matrix. Five characteristic features are processed in the proposed system, i.e. entropy, contrast, homogeneity, energy, and correlation. Entropy is used to measure the randomness of intensity distributions. The entropy value is calculated by:

$$
\text { Entropy }=-\sum_{i=0}^{I} \sum_{j=0}^{J} p(i, j) \log p(i, j)
$$

For taking into account the power of intensity differences in an image, the contrast characteristic is evaluated. The contrast value is calculated by:

$$
\text { Contrast }=\sum_{i=0}^{I} \sum_{j=0}^{J}(i-j)^{2} p(i, j)
$$

The homogeneity which calculates the uniformity of intensity variations in an image, is the contrary of the image contrast. Below is the equation for calculating the homogeneity:

$$
\text { Homogeneity }=\sum_{i=0}^{I} \sum_{j=0}^{J} \frac{p(i, j)}{1+|i-j|}
$$

Next, energy, as the fourth features, is used to measure the texture uniformity. The energy value is calculated by:

$$
\text { Energy }=\sum_{i=0}^{I} \sum_{j=0}^{J}(P(i, j))^{2}
$$

Finally, the correlation value is used to describe the relations between each pixel value with its neighbors. The correlation value is calculated by:

$$
\text { Correlation }=\frac{\sum_{i=0}^{i} \sum_{j=0}^{j}[(i j) p(i, j)]-\mu x \mu y}{\sigma x \sigma y}
$$

\section{EXPERIMENTS}

This section describes the experiments conducted for the proposed leukocyte detection and recognition system using the Scale Invariant Feature Transform method. We developed our own database, called the FTI-Untar blood cells database. The FTI-Untar blood cells database consists of a total of 183 blood cell images with 112 neutrophils images, 37 lymphocytes images, 21 monocytes images, 10eosinophils images, and 3 basophils images. The images were taken using a digital camera with $1600 \times 1200$ pixels that was attached to a microscope. Fig. 2 shows the sample of a blood cell image that is used in the experiment, whileFig. 3 shows the samples of blood cell images, the cropped leukocyte images, the cropped leukocyte images with $10 \%$ darken lighting effects, and the cropped leukocyte images with $10 \%$ brighten lighting effects.

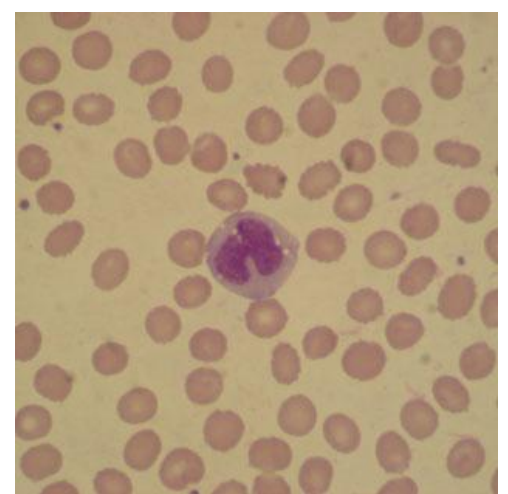

Fig. 2. The sample of a blood cell image for leukocyte detection system.

We have conducted various experiments with various targets for the proposed leukocyte detection and recognition system. First, we evaluated the performance of the SIFT method for detecting the white blood cells. Table I shows the detection results using SIFT method. It is shown in Table I that the proposed system was able to detect white blood cells with more than $86.67 \%$ accuracy.

TABLE I: THE DETECTION RESULTS USING SIFT METHOD

\begin{tabular}{ccccc}
\hline $\begin{array}{c}\text { Blood Cell } \\
\text { Type }\end{array}$ & $\Sigma$ data & ¿training & $\begin{array}{c}\Sigma \\
\text { testing }\end{array}$ & $\begin{array}{c}\text { Accuracy } \\
(\%)\end{array}$ \\
\hline Neutrophil & 112 & 67 & 45 & 86.67 \\
Eosinophil & 10 & 6 & 4 & 100 \\
Basophil & 3 & 2 & 1 & 100 \\
Lymphocyte & 37 & 22 & 15 & 86.67 \\
Monocyte & 21 & 12 & 9 & 100 \\
\hline
\end{tabular}

TABLE II: THE DETECTION RESUlts USING SIFT METHOD FOR DARKEN

\begin{tabular}{ccccc}
\multicolumn{5}{c}{ LIGHTING EFFECTS } \\
\hline Blood Cell & $\Sigma$ data & \multicolumn{3}{c}{ Accuracy (\%) } \\
\cline { 3 - 5 } Type & & Natural & Darken & Darken \\
& & Lighting & $5 \%$ & $10 \%$ \\
\hline Neutrophil & 112 & 86.67 & 82.22 & 80 \\
Eosinophil & 10 & 100 & 100 & 75 \\
Basophil & 3 & 100 & 100 & 100 \\
Lymphocyte & 37 & 86.67 & 86.67 & 80 \\
Monocyte & 21 & 100 & 100 & 88.89 \\
\hline
\end{tabular}

In the next experiments, we tested the system with various lighting conditions. Table II and Table III show the detection 
results using SIFT method for images with various darken and brighten lighting conditions, respectively. The natural lighting means the condition where images were taken with microscope standard lighting, while darken and brighten effects were done by adjusting the contrast of the natural lighting images using a picture editing software. As shown in Table II, the detection accuracies for images with darken lighting effects were lower than that of with natural lighting. However, the detection accuracies were still higher than $75 \%$. Meanwhile, the detection accuracies for images with brighten lighting effects were lower than that of the natural lighting and the darken effects. However, the detection accuracies were still higher than $75 \%$ as shown in Table III.

TABLE III: THE DETECTION RESULTS USING SIFT METHOD FOR BRIGHTEN LIGHTING EFFECTS

\begin{tabular}{ccccc}
\hline $\begin{array}{c}\text { Blood Cell } \\
\text { Type }\end{array}$ & $\Sigma$ data & \multicolumn{3}{c}{ Accuracy (\%) } \\
\cline { 3 - 5 } & & $\begin{array}{c}\text { Natural } \\
\text { Lighting }\end{array}$ & $\begin{array}{c}\text { Brighten } \\
5 \%\end{array}$ & $\begin{array}{c}\text { Brighten } \\
10 \%\end{array}$ \\
\hline Neutrophil & 112 & 86.67 & 84.44 & 75.56 \\
Eosinophil & 10 & 100 & 100 & 75 \\
Basophil & 3 & 100 & 100 & 100 \\
Lymphocyte & 37 & 86.67 & 86.67 & 86.67 \\
Monocyte & 21 & 100 & 100 & 88.89 \\
\hline
\end{tabular}

We also tested various threshold values for keypoint localizations in SIFT method. Table IV shows the detection results using SIFT method with three different threshold values: $0.02,0.03$, and 0.05 . It is clearly seen that the detection accuracies using $\theta=0.03$ gave the highest results compared to the other threshold values.

Finally, we conducted experiments to recognize leukocyte types from images which have fixed window sizes, both for training and testing images, i.e. $47 \times 47$ pixels and $57 \times 57$ pixels. These dimensions were the average size of leukocyte cells that were captured from the microscopic images. The recognition accuracies for images with various cropping sizes are shown in Table V. We applied two recognition methods for the leukocyte recognition system: 1) the Euclidean Distance method and 2) the Co-occurrence Matrix method. Based on Table $\mathrm{V}$, the overall recognition accuracies for images with $47 \times 47$ pixels and $57 \times 57$ pixels sizes with the Euclidean Distance method were $82.79 \%$ and $84.93 \%$, respectively. Meanwhile, the overall recognition accuracy for leukocyte recognition system using the Co-occurrence Matrix method was $75.93 \%$ for images with $47 \times 47$ pixels and $61.78 \%$ for images with $57 \times 57$ pixels sizes. In general, for both recognition methods, it is clearly seen that Basophilwas the most difficult leukocyte type to find and to recognize.

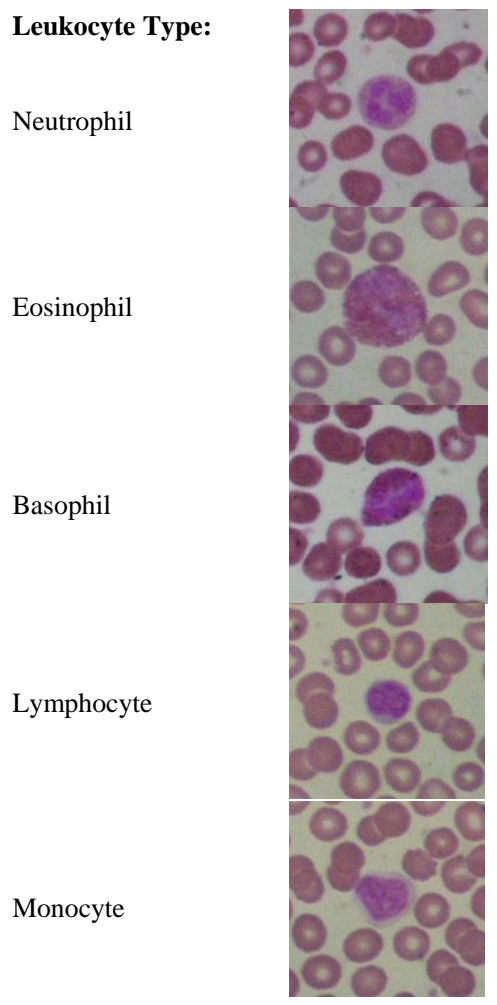

Blood cell images

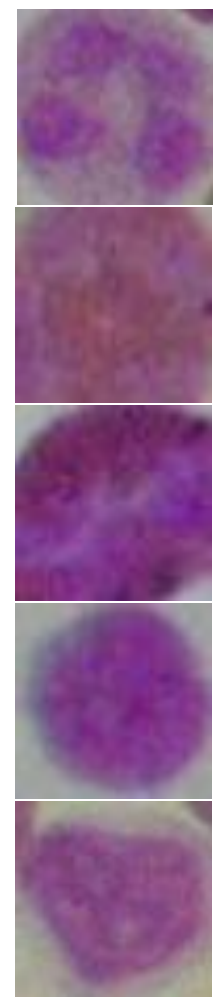

Cropped leukocyte images

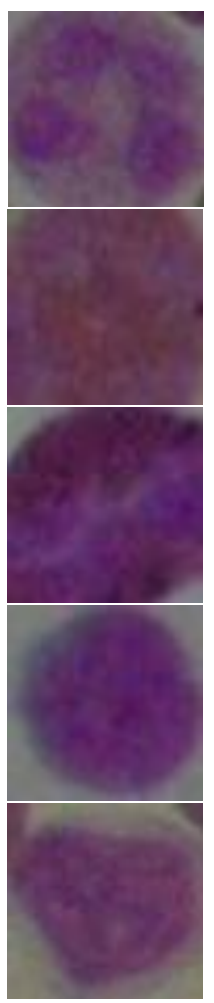

Cropped

leukocyte image with $10 \%$ darken lighting effects

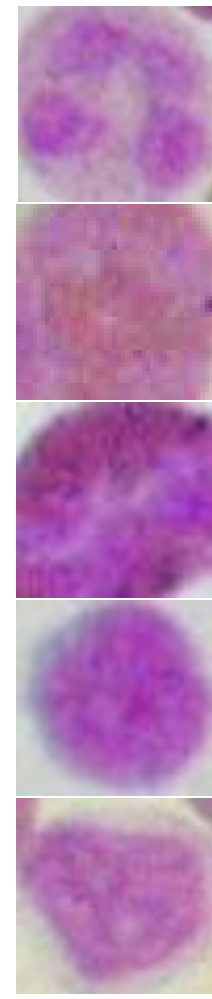

Cropped

leukocyte images with $10 \%$ brighten lighting effects

Fig. 3. The image samples of white blood cells used in the experiments.

TABLE IV: THE DETECTION RESUlTS USING SiFT METHOD WITH VARIOUS THRESHOLD VALUES FOR KEYPOINT LOCALIZATION

\begin{tabular}{|c|c|c|c|c|c|c|}
\hline \multirow[t]{2}{*}{ Type } & \multirow[t]{2}{*}{ Edata } & \multirow[t]{2}{*}{ Straining } & \multirow[t]{2}{*}{ Etesting } & \multicolumn{3}{|c|}{ Accuracy (\%) } \\
\hline & & & & $\theta=0.02$ & $\theta=0.03$ & $\theta=0.05$ \\
\hline Neutrophil & 112 & 67 & 45 & 77.78 & 86.67 & 93.33 \\
\hline Eosinophil & 10 & 6 & 4 & 50 & 100 & 75 \\
\hline Basophil & 3 & 2 & 1 & 0 & 100 & 100 \\
\hline Lymphocyte & 37 & 22 & 15 & 66.67 & 93.33 & 93.33 \\
\hline Monocyte & 21 & 12 & 9 & 88.89 & 100 & 100 \\
\hline
\end{tabular}


TABLE V: THE RECOGNITION RESULTS FOR LEUKOCYTE IMAGES WITH VARIOUS CROPPING SIZES

\begin{tabular}{|c|c|c|c|c|c|c|}
\hline \multirow{3}{*}{ Type } & \multirow{3}{*}{ ¿training } & \multirow{3}{*}{ Etesting } & \multicolumn{4}{|c|}{ Accuracy (\%) } \\
\hline & & & \multicolumn{2}{|c|}{ Image size $47 \times 47$ pixels } & \multicolumn{2}{|c|}{ Image size $57 \times 57$ pixels } \\
\hline & & & $\begin{array}{c}\text { Euclidean } \\
\text { Distance }\end{array}$ & $\begin{array}{l}\text { Co-occurrence } \\
\text { Matrix }\end{array}$ & $\begin{array}{l}\text { Euclidean } \\
\text { Distance }\end{array}$ & $\begin{array}{l}\text { Co-occurrence } \\
\text { Matrix }\end{array}$ \\
\hline Neutrophil & 213 & 53 & 90.62 & 73.58 & 96.88 & 90.56 \\
\hline Eosinophil & 100 & 22 & 83.33 & 63.63 & 77.78 & 59.09 \\
\hline Basophil & 74 & 20 & 40 & 55 & 50 & 30 \\
\hline Lymphocyte & 10 & 3 & 100 & 66.67 & 100 & 100 \\
\hline Monocyte & 3 & 2 & 100 & 50 & 100 & 100 \\
\hline
\end{tabular}

\section{CONCLUSION}

We have presented the Scale Invariant Feature Transform method to automatically detect leukocyte areas and recognize the leukocyte types from microscopic images. The detection results of the leukocyte images using SIFT method are highly dependent on the threshold value of keypoint localization. Other parameters such as lighting condition and window size also give significant effects on the accuracy of the system. For the recognition system, the Euclidean Distance method gives a slightly better result than that of the color based recognition, i.e. the Co-occurrence Matrix method.

In the future, we consider to develop a dynamic window model for detecting the leukocyte area, the use of other color domains, i.e. Hue, Saturation, and Value (HSV) for improving the system's accuracy.

\section{REFERENCES}

[1] L. A. Chris and B. Mulyawan, "Focused color intersection for leukocyte detection and recognition system," International Journal of Information and Electronics Engineering, vol. 3, no. 5, pp. 498-501, Sep. 2013.

[2] T. Markiewicz, "Data mining techniques for feature selection in blood cell recognition," in Proc. European Symposium on Artificial Neural Networks, 2006, pp. 407-412.

[3] M. C. Colunga, O. S. Siordia, and S. J. Maybank, "Leukocyte Recognition using EM-algorithm," in Proc. 8th Mexican International Conference on Artificial Intelligence, 2009, pp. 545-555.

[4] N. T. Umpon and P. D. Gader, "Training neural networks to count white blood cells via a minimum counting error objective function," in Proc. International Conference on Pattern Recognition, 2000, pp. 2299-2302.

[5] M. Beksac, M. S. Beksac, V. B. Tipi, H. A. Duru, M. U. Karakas, and A. Cakar, "An artificial intelligent diagnostic system on differential recognition of hematopoietic cells from microsopic images", Cytometry, vol. 30, pp. 145-150, Jun. 1997.

[6] D. G. Lowe, "Object Recognition from Local Scale-Invariant Features," in Proc. International Conference on Computer Vision, 1999, pp. 1150-1157.

[7] D. G. Lowe, "Local Feature View Clustering for 3D Object Recognition," in Proc. IEEE Conference on Computer Vision and Pattern Recognition, 2001, pp. 682-688.

[8] D. G. Lowe, "Distinctive image features from scale-invariant key-points," International Journal of Computer Vision, vol. 60, no. 2, pp. 91-110, Feb. 2004.

[9] F. Alhwarin, C. Wang, D. R-Durrant, A. Graser, "Improved SIFT-Features Matching for Object Recognition," in Proc. BCS International Academic Conference, 2008, pp. 179-190.

[10] C. Harris and M. Stephens, "A combined corner and edge detector," in Proc. the 4th Alvey Vision Conference, 1988, pp. 147-151.

[11] J. Chen, L-H. Zou, and L-H. Dou, "The comparison and application of corner detection algorithms," Journal of Multimedia, vol. 4, no. 6, pp. 435-441, Dec. 2009.
[12] L. A. Chris and B. Mulyawan, "A combination of feature selection and co-occurrence matrix methods for leukocyte recognition system," Journal of Software Engineering and Applications, vol. 5, no. 12B, pp. 101-106, Dec. 2012.

[13] A. Chris, S. Sugiharto, and Lina, "Detection of Abnormalities of Lymph Node Tissues using Image Texture Analysis," in Proc. International Conference on Information Technology and Applied Mathematics, 2012, pp. 30-32.

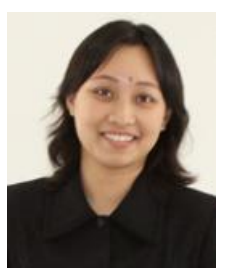

Lina Arlends Chris was born in Padang, West Sumatera, Indonesia in 1979. She received a B.Eng. degree in computer science from Tarumanagara University, Indonesia in 2001, and a M.Sc. degree in computer science from the University of Indonesia in 2004. In 2005, she received the Japanese government scholarship as a research student at the Graduate School of Information Science, Nagoya University, Japan. Later she continued her graduate study and got her doctoral degree from Nagoya University, Japan in 2009. Currently she is working as a lecturer at Tarumanagara University, Indonesia. Her research interest is in pattern recognition and computer vision field. She is also a member of IEEE and IEICE.

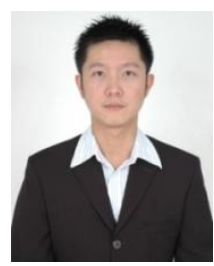

Arlends Chris was born in Bogor, West Java, Indonesia on March 21, 1977. He got his medical doctor degree from the Faculty of Medicine from Tarumanagara University, Indonesia, in 2005. Later, he obtained his master degree in educational psychology from Tarumanagara University in 2009. Now, he is working as a lecturer at the Faculty of Medicine, Tarumanagara University. He is also a member of the Indonesian Medical Association.

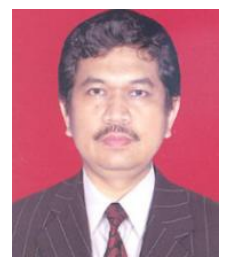

Bagus Mulyawan was born in Yogyakarta, Central Java, Indonesia in 1962. He got his bachelor degree from the Computer Engineering Department, Gunadarma University, Indonesia in 1992. He obtained his master degree in information system management from Budi Luhur University, Jakarta, Indonesia in 2008. Now, he is working as a lecturer at the Faculty of Information Technology, Tarumanagara

University.

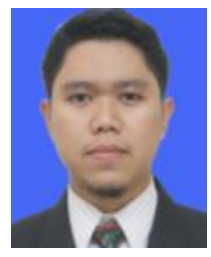

Agus Budi Dharmawan received the MSc degree from the Department of Electrical Engineering, Fachbereich EIT, University of Applied Sciences Hochschule Darmstadt(hda) Germany in 2011 and the MT degree from the Department of Electrical Engineering, ITS Surabaya, Indonesia in 2011. He has been with the Tarumanagara University, Jakarta, Indonesia since 2008. His research interests include robotics, computer vision, machine learning, and pattern recognition 